\title{
Mortalidad por infarto del miocardio en Chile. Trombolíticos o angioplastía
}

\author{
FERNANDO FLORENZANO U.
}

\section{Acute myocardial infarction mortality in Chile. Thrombolysis or angioplasty}

During recent decades, acute myocardial infarction short-term mortality has decreased to one digit levels, in the United States. Data from Chilean registries give figures around $11 \%$ for patients receiving thrombolysis, and 5 to $6 \%$ for patients treated with angioplasty. The decrease in mortality in Chile is related to the implementation of the AUGE program at a national level, initiative than gives patients the opportunity to receive at least thrombolytic therapy as well as a standardized and rapid diagnosis and treatment for this condition. There is a lack of Outcome Research studies in Chile, that would guide public health decisions such as the use of fibrin-specific agents for early presentation and high risk cases, and a rational system providing better access to primary angioplasty.

(Rev Med Chile 2011; 139: 1393-1395).

Key words: Mortality; Myocardial infarction; Myocardial reperfusion; Thrombolytic therapy.

4 n las últimas décadas, la mortalidad intrahospitalaria por infarto agudo del miocardio con supradesnivel de ST ha descendido desde cifras cercanas a 30\% (antes de la era de las Unidades Coronarias), a cifras menores del 10\% , en Norteamérica ${ }^{1-2}$. Inicialmente, este descenso se debió a la mejor posibilidad de reconocer y tratar precozmente complicaciones como arritmias y falla de bomba; posteriormente, se reconoció la importancia de una reperfusión precoz y se implementaron las técnicas para llevarla a cabo: uso de trombolíticos y de angioplastía primaria -con balón, inicialmente, y luego con el uso de "stents"-. En años recientes, en nuestro país se habían publicado dos trabajos observacionales que compararon la eficacia relativa de ambas estrategias de reperfusión: el año 2008 el grupo GEMI comunicó la mortalidad de los tres centros de Santiago que aportaron más pacientes a ese registro: $10,9 \%$ para trombolisis versus $5,6 \%$ para angioplastia, en su evolución intrahospitalaria, diferencia que persiste como significativa luego de ajustar por covariables relevantes de riesgo, y en la evaluación alejada ${ }^{3}$. Más recientemente, el
2010, se comunicó la comparación de un grupo mayor de pacientes, aportados por los registros GEMI y RENAC, con resultados muy semejantes ${ }^{4}$. A estas estimaciones de mortalidad por infarto en Chile, se agregan los datos del estudio publicado en esta edición por Ugalde et al, datos de un solo centro en Santiago, comunicando mortalidad intrahospitalaria de $11,2 \%$ para trombolisis, y de $2 \%$ para angioplastía ${ }^{5}$. Estas últimas cifras resultan más difíciles de aceptar como una estimación válida de la realidad nacional, por constituir un grupo menos numeroso de pacientes y por haber excluido los pacientes más graves, sin que los autores revelen el número de tales exclusiones, ni el tratamiento preferente de reperfusión en ese grupo. En general, es necesario reconocer que los datos de mortalidad intrahospitalaria provenientes de registros probablemente subestiman la cifra representativa de un país, en un momento determinado, entre otros motivos por provenir los datos de centros en los cuales existen protocolos de diagnóstico y tratamiento precoces instalados desde hace tiempo. Una observación epidemiológica reciente de Nazzal y Alonso esti- 
man la letalidad intrahospitalaria del infarto del miocardio en Chile en 14,2\%, observándose una disminución entre los años 2001 y $2007^{6}$.

Hoy es ampliamente aceptado que, para la mayor parte de los pacientes que se presentan a un centro asistencial con un infarto, la angioplastía es una estrategia más eficaz de reperfusión que la trombolisis -en el supuesto que ambas técnicas estén disponibles- aún considerando el uso de trombolíticos fibrino específicos, poco usados en nuestro país. Este consenso se consolidó progresivamente al conocerse los resultados de ensayos clínicos que compararon ambas técnicas de reperfusión y de los metanálisis que conglomeraron esos datos; éstos últimos revelan una ventaja de mortalidad y de ocurrencia de eventos indeseables como hemorragias, favorable para la angioplastía ${ }^{7}$. Habiéndose demostrado la superioridad de la angioplastía ¿tiene sentido continuar la exploración del tema en base a datos provenientes de registros? En mi opinión sí, puesto que estos últimos pueden dar cuenta de la práctica médica -en el mundo real-, lo que muchos ensayos clínicos no hacen al imponer estrictos criterios de inclusión y exclusión. Sería deseable, sin embargo, que los estudios provenientes de registros tuviesen amplitud suficiente para cubrir experiencias en distintos tipos de centros asistenciales; en lo posible, deberían contar con análisis de costo efectividad; solo entonces podrán constituir verdaderos aportes al planeamiento de las acciones de salud, configurando el tipo de investigación que se ha llamado 'Outcomes Research'.

En este sentido, un aporte válido de este tipo de investigación centrada en la efectividad de los resultados de intervenciones en el sentido más amplio de la palabra, lo constituyó un análisis del grupo GEMI sobre el impacto del plan AUGE en nuestro país, en el tratamiento del infarto: estos investigadores compararon una cifra importante de pacientes tratados en 10 centros de regiones y de Santiago, antes y después de la implementación del plan de garantías explicitas, en el cual se provee con una importante cobertura de trombolisis con estreptoquinasa -como estándar mínimo en la estrategia de reperfusión- además de proporcionar el resto del tratamiento coadyuvante. Este estudio mostró una fuerte asociación entre la implementación del AUGE y la disminución de la mortalidad por infarto. ${ }^{8}$

¿Qué información sería necesaria en un país como el nuestro para avanzar en este importante tema? Deberíamos contar con estudios de costo efectividad para decidir algunas posibles reformas al sistema de atención del infarto: en algunos países desarrollados, se ha generalizado el uso de trombolíticos fibrino específicos frente a toda situación en la cual no sea factible la angioplastía primaria inmediata o luego de un traslado rápido a un centro con capacidad para efectuarla. En otros países, en tanto, se ha estimado que el costo de esta estrategia es prohibitivo y que la ventaja del rendimiento de los trombolíticos fibrino específicos comparada con la estreptoquinasa no se justifica para el tratamiento masivo. Se ha propuesto el uso de agentes con mayor tasa de reperfusión que la estreptoquinasa en algunas situaciones, como pacientes de mayor riesgo, o bien frente a la presentación muy precoz. Otro aspecto que debería investigarse con estudios de costo efectividad se refiere a cuánto es razonable invertir para ampliar la cobertura de la angioplastia primaria: si bien su superior eficacia comparada con trombolisis está comprobada, su efectividad se resiente al considerar su cobertura limitada a las grandes urbes y a su costo considerablemente mayor, en comparación con la trombolisis con estreptoquinasa. Otros países han invertido fuertemente en sistemas de alerta, de asignación de cupos de laboratorio invasivo y de traslado eficiente. Para poder efectuar recomendaciones válidas a la autoridad sanitaria deberíamos contar con investigación de resultados de alta calidad.

\section{Referencias:}

1. Braunwald E. Heart Disease: A Textbook of Cardiovascular Medicine. 5a Edición. Philadelphia. 1997, p. 1185.

2. Yeh R, Sidney S, Chandra M, Sorel M, Go A. Population Trends in the Incidence and Outcomes of Acute Myocardial Infarction. N Engl J Med 2010; 362: 2155-65.

3. Greig D, Corbalán R, Castro P, Lamich R, Yovaniniz P. Impacto de la trombolisis y de la angioplastía primaria en pacientes con infarto agudo del miocardio tratados en centros hospitalarios terciarios. Rev Med Chile 2008; 136: 1098-1106.

4. Martínez A, Nazzal C, Fajuri A, Barra L, Mayerson A, Cavada G, et al. Mortalidad post infarto del miocardio en Chile: comparación de los registros de angioplastía primaria versus trombolisis. Rev Chil Cardiol 2010; 29: 29-36. 
5. Ugalde H, Ugalde D, Muñoz M. Terapia de reperfusión en infarto agudo al miocardio. Comparación entre trombolisis endovenosa y angioplastía coronaria. Rev Med Chile 2011; 139: 1396-1402.

6. Nazzal C, Alonso F. Incidencia y letalidad por infarto agudo del miocardio en Chile: 2001-2007. Rev Med Chile 2011; 139: 1253-60.

7. Keeley E, Boura J, Grines C. Primary angioplasty versus intravenous thrombolytic therapy for acute myocardial infarction. A quantitative review of 23 randomyzed trials. Lancet 2003; 361: 13-20.

8. Nazzal C, Campos P, Corbalán R, Lanas F, Bartolucci J, Sanhueza C, et al. Impacto del Plan AUGE en el tratamiento de pacientes con infarto agudo al miocardio con supradesnivel de ST, en hospitales chilenos. Rev Med Chile 2008; 136: 1231-9. 